\title{
REVIEW
}

\section{The evolutionary ecology of complex lifecycle parasites: linking phenomena with mechanisms}

\begin{abstract}
SKJR Auld and MC Tinsley
Many parasitic infections, including those of humans, are caused by complex lifecycle parasites (CLPS): parasites that sequentially infect different hosts over the course of their lifecycle. CLPs come from a wide range of taxonomic groups-from single-celled bacteria to multicellular flatworms-yet share many common features in their life histories. Theory tells us when CLPs should be favoured by selection, but more empirical studies are required in order to quantify the costs and benefits of having a complex lifecycle, especially in parasites that facultatively vary their lifecycle complexity. In this article, we identify ecological conditions that favour CLPs over their simple lifecycle counterparts and highlight how a complex lifecycle can alter transmission rate and trade-offs between growth and reproduction. We show that CLPs participate in dynamic host-parasite coevolution, as more mobile hosts can fuel CLP adaptation to less mobile hosts. Then, we argue that a more general understanding of the evolutionary ecology of CLPs is essential for the development of effective frameworks to manage the many diseases they cause. More research is needed identifying the genetics of infection mechanisms used by CLPs, particularly into the role of gene duplication and neofunctionalisation in lifecycle evolution. We propose that testing for signatures of selection in infection genes will reveal much about how and when complex lifecycles evolved, and will help quantify complex patterns of coevolution between CLPs and their various hosts. Finally, we emphasise four key areas where new research approaches will provide fertile opportunities to advance this field.
\end{abstract}

Heredity (2015) 114, 125-132; doi:10.1038/hdy.2014.84; published online 17 September 2014

\section{INTRODUCTION}

Parasites vary in the complexity of their lifecycles. Some complete their lifecycle in a single host (simple lifecycle parasites, SLPs), while others require multiple successive hosts (complex lifecycle parasites, CLPs); some parasitic helminths must sequentially infect four obligate host species to complete their lifecycle (Cribb et al., 2003). CLPs are of substantial applied importance because they cause severe diseases of humans and livestock, including malaria, toxoplasmosis, river blindness, Lyme disease, schistosomiasis and sleeping sickness. However, despite abundant theory (Choisy et al., 2003; Parker et al., 2003; Gandon, 2004; Iwasa and Wada, 2006) and the clear relevance to human health, we lack empirical studies of the evolutionary causes and consequences of parasite lifecycle complexity.

At first glance, the evolutionary transition from simple to complex lifecycle seems unlikely, as it requires the parasite to rely on multiple host species, usually in a particular sequence, in order to complete its lifecycle. Nevertheless, CLPs abound in nature and have evolved in multiple disparate taxa, including bacteria (for example, Borrelia; Pal and Fikrig, 2003), flatworms (Neodermata: Schistosoma, Taenia; Park et al., 2007), roundworms (Nematoda; Anderson, 2000), acanthocephalan worms (Acanthocephala; Crompton and Nickol, 1985), Arthropods (Pentastomida; Riley, 1983) and two different lineages of protists: the Apicomplexa (which includes Plasmodium, Toxoplasma and Babesia; Roos, 2005), and Kinetoplastida (Trypanosoma; Vickerman et al., 1988; Tyler and Engman, 2001).
In this review, we examine conditions that favour complexity, the immediate benefits of a complex lifecycle, its long-term (co)evolutionary consequences and the evolution of mechanisms that permit infection of multiple successive hosts. We propose testable hypotheses for both why and how parasites evolved a complex lifecycle, and emphasise the need for more empirical studies that quantify the costs and benefits of increased lifecycle complexity.

\section{EVOLVING A COMPLEX LIFE CYCLE}

Parker et al. (2003) suggested two mechanisms that may have led helminths to divide their lifecycles across hosts. The first of these is upward incorporation (UI) and requires the presence of predators that routinely eat (and sometimes actively choose) parasite-infected prey (for example, Orlofske et al., 2012). If parasites can adapt to survive and reproduce in the predators of their hosts, they may acquire the predator as a second host. The second mechanism is downward incorporation (DI). DI evolves in two stages: first, a directly transmitted parasite evolves the capability to survive independently of its host; and second, the parasite evolves to infect a second host species that routinely ingests these parasite transmission stages, thus acquiring a second host (Parker et al., 2003). UI and DI can both, for different reasons, lead to increased parasite fitness: UI permits a longer parasite lifespan, greater body size and increased fecundity, whereas DI leads to reduced mortality of parasite propagules and therefore increased probability of transmission (Parker et al., 2003). 
There are, however, constraints to evolving complexity. Choisy et al. (2003) demonstrated that selection should only favour an increase in lifecycle complexity when intermediate hosts are more abundant than the definitive host, parasite survival in the intermediate host is high and transmission from the intermediate host to the definitive host is common (that is, when lifecycle complexity increases the efficiency of parasite transmission). Increased lifecycle complexity should evolve only if the cost of being able to infect multiple hosts is low (Choisy et al., 2003). Gandon's (2004) work supports these findings: after designing models with a malaria system in mind, he found that indirect vector-borne transmission was favoured when the supply of vectors was high compared with the supply of the definitive host.

The benefits of a complex lifecycle must be realised rapidly for it to be favoured by selection. Morand et al. (1995) tested for these benefits by comparing the fitness of two closely related parasitic cestodes that differed in the number of intermediate hosts they infect. Bothriocephalus barbatus infects a marine copepod as its intermediate host and a flatfish (Scopthalmus rhombus) as its definitive host. Bothriocephalus gregarious infects the copepod as a first intermediate host, gobies (Pomatoschistus sp.) as second intermediate paratenic (non-essential) hosts and a different flatfish, (Psetta maxima) as its definitive host (Robert et al., 1988). Although not strictly necessary for the parasite to complete its lifecycle, gobies likely benefit $B$. gregarious infectious stages by increasing overall transmission: the total prevalence of $B$. gregarious is higher than that of B. barbatus (Morand et al., 1995). However, although comparing two closely related parasite species with different levels of complexity is useful, one cannot isolate the effect of differing lifecycle complexity from other unknown phenotypic influences on parasite transmission potential; $B$. barbatus and B. gregarious are different in many respects in addition to the complexity of their lifecycles, for example, the identity of their definitive hosts. How can this problem be overcome? Parasites that facultatively alter the number of hosts they infect provide an excellent opportunity to test the immediate benefits of complexity, as one can easily examine relationships between lifecycle complexity, parasite transmission and parasite fitness in a single parasite species. The trematode, Coitocaecum parvum is an example of such a parasite.

Coitocaecum parvum can adopt a three-host (snail-amphipod-fish) or a two-host (snail-amphipod) lifecycle. Once in the haemocoel of the amphipod host (Paracalliope fluviatilis), C. parvum can either await ingestion by the fish Gobiomorphus cotidianus where it sexually reproduces in its gut (three-host lifecycle), or continue to grow and then reproduce by selfing in the amphipod (two-host lifecycle; Lagrue and Poulin, 2007; Lagrue et al., 2007). Coitocaecum parvum only switches to a three-host lifecycle in the presence of the third (fish) host
(Poulin, 2003). Although the three-host lifecycle allows crossfertilisation and is therefore probably optimal (outbreeding provides immediate fitness benefits), the two-host strategy acts as a form of reproductive insurance, allowing the parasite to complete its lifecycle in the absence of the third host and thus avoid death (Lefebvre and Poulin, 2005, but see Wang and Thomas, 2002).

\section{THE TRIALS OF TRANSMISSION}

CLPs face the challenge of ensuring transmission between multiple host species to complete their lifecycle, and failure at any one of these transmission events could result in the death of the parasite. Parasites that can facultatively vary the number of hosts they infect (for example, C. parvum) raise many interesting questions. When such parasites are restricted to a simpler lifecycle for multiple generations, does the fitness loss associated with inbreeding accumulate? Does sustained selection on a two-host lifecycle lead to evolutionary changes in virulence (on any of the hosts) or between-host transmission?

There are abundant examples where transmission constraints have selected on CLPs to revert to a simpler (and easier to complete) lifecycle. Various trematode species have given up their definitive host (either facultatively or completely; Poulin and Cribb, 2002; Lagrue et al., 2007), evolved to use a second intermediate host as a definitive host (Grabda-Kazubska, 1976), or have foregone paratenic hosts (Nasincova and Scholz, 1994). Poulin and Cribb (2002) note 32 trematode families have reduced lifecycles and that lifecycle truncation has occurred on at least 20 occasions in the evolutionary history of the Trematoda. Life cycle truncation is, however, often accompanied with other major life history changes - the apicomplexan Toxoplasma gondii provides a prime example.

Toxoplasma can establish infections in almost any endothermic vertebrate as an intermediate host and undergo asexual replication, but for the parasite to reproduce sexually, it must infect a felid (the definitive host; Dubey, 1977). However, some T. gondii lineages have reverted to a simple lifecycle without a felid definitive host, and have forgone sexual reproduction as a consequence ( $\mathrm{Su}$ et al., 2003). It remains to be determined whether this switch was initially favoured by selection because of limited opportunities for transmission to felids. Lifecycle truncation has resulted in the massive clonal expansion of three asexual $T$. gondii lineages, and has been implicated in increased pathogenicity to humans (Su et al., 2003). Does a loss of sex involve increased asexual replication, and if so, does this explain the increased pathogenicity of asexual strains (Su et al., 2003)? Long-term experimental evolution studies could effectively address these questions in controlled laboratory environments (see Box 1), and whole-genome sequencing studies comparing sexual and asexual T. gondii strains

\section{Box 1 Four key research themes to understand parasites with complex lifecycles.}

We suggest the following avenues of research will strengthen our understanding of both the causes and consequences of increased lifecycle complexity in parasites:

(1) Conduct experimental evolution studies using parasites that can facultatively vary the complexity of their lifecycle and examine how increased lifecycle complexity impacts on parasite transmission rate and virulence.

(2) Test the hypothesis that dividing growth and reproduction according to host type is advantageous to CLPs by experimentally manipulating the genetic diversity of infections in intermediate and definitive hosts and measuring long-term consequences for parasite fitness traits.

(3) Study the relationship between relative migration rate of host types and patterns of parasite LA across various taxa of CLPs. Test the hypothesis that more mobile hosts will fuel parasite LA to less mobile hosts.

(4) Examine sequence variation and expression differences in genes associated with infecting intermediate and definitive hosts. Test the hypothesis that gene duplication events followed by neofunctionalisation in infection genes are associated with the evolution of a complex lifecycle. 
(such as that by Minot et al., 2012) would provide an opportunity to identify the evolutionary changes underlying this marked lifecycle modification.

\section{Parasite manipulation of host behaviour}

As an alternative to lifecycle truncation, constraints can select for CLP manipulation of an intermediate host's behaviour to increase transmission to the definitive host. However, infection-induced changes in host behaviour may not always be adaptive parasite strategies; they could be mere side effects of infection (for example, reduced motility) and could increase the likelihood that infected hosts are, for example, consumed by other predators in which the parasite cannot complete its lifecycle (Poulin, 1995). The most convincing evidence that manipulation of host behaviour is adaptive to the parasite comes from demonstrations that the parasite (1) only induces a behavioural change at a specific time that favours its own fitness; and (2) this behavioural change increases the likelihood of transmission to a definitive host in which the parasite can complete its lifecycle. This has been examined in numerous studies. We provide a few key examples below.

Using a simple optimality model, Hammerschmidt et al. (2009) determined an optimal duration of infection for the tapeworm Schistocephalus solidus in its intermediate host (the copepod, Macrocyclops albidus). They then tested their model experimentally by altering the duration of infection in the copepod, and found immature S. solidus actively suppressed copepod activity, reducing the likelihood of premature fish predation (and death of the immature parasites); however, when $S$. solidus reached the next developmental stage and required transmission to the second intermediate (fish) host, they increased copepod activity and the likelihood of predation (Hammerschmidt et al., 2009). Similarly, Dianne et al. (2012) demonstrated that the Amphipod, Gammarus pulex, exhibited stronger anti-predator behaviour (that is, was more likely to hide in predator refuges) when infected with immature stages of the acanthocephalan Pomphorhynchus laevis, than when uninfected. Once P. laevis had reached maturity within its $G$. pulex host, this anti-predator behaviour decreased and infected G. pulex were significantly more likely to experience predation by the definitive host, the brown trout, Salmo trutta. There is also compelling evidence that T. gondii manipulates the behaviour of its intermediate rat hosts to make them sexually attracted to the odour of cats, the definitive host (House et al., 2011).

\section{THE LIFE HISTORY TRADE-OFFS FACED BY CLPS}

Like most organisms, parasites often face a trade-off between growth and reproduction because environments that are optimal for growth may be suboptimal for reproduction and vice versa. Parasite growth (or asexual replication) is often lower when within-host competition is high (for both SLPs and CLPs). CLP coinfections (multi-genotype infections) in intermediate hosts occur in many but not all helminth taxa, and when they do occur (either naturally or experimentally), competition leads to clear parasite fitness reductions (De Roode et al., 2005; Bell et al., 2006; Jäger and Schjørring, 2006; Michaud et al., 2006; Lagrue and Poulin, 2008; Balmer et al., 2009; Cornet, 2011; Dianne et al., 2012). However, sexually reproducing parasites often benefit from being in genotypically diverse infections, because they can acquire genetically dissimilar mates (Brown et al., 2001) and thus reduce the incidence of inbreeding and the associated fitness costs. Even for CLPs that can self-fertilise, cross-fertilisation is often favourable (Christen and Milinski, 2003) and may even be required on some occasions in order for the parasite to maintain offspring viability and prevent a decline in overall fecundity (Wedekind et al.,
1998). In definitive hosts, the benefits of outbreeding may outweigh the costs associated with within-host competition. Below, we outline a hypothesis that suggests CLPs may, in the short-term, be less constrained by the growth-reproduction trade-off than their SLP counterparts.

\section{Short-term escape from the growth-reproduction trade-off}

Here, we argue that a complex lifecycle may be favoured by selection because it can reduce or even eliminate the growth-reproduction trade-off (at least in the short term) by allowing the parasite to separate growth and reproduction according to host type. As Figure 1 shows, a CLP can undergo growth in intermediate hosts alongside few (if any) other genotypes, and then reproduce in definitive hosts, where there may be many parasite genotypes. Indeed, both the intensity (measured as parasite burden) and genetic diversity of infections tend to be higher in definitive hosts compared with intermediate hosts, because of concentration of parasites up the food chain (Morand et al., 1995; Brown et al., 2001). For example, in the trematode Diplostomum pseudopathaceum, a small number of parasite genotypes (usually just one) establish infections in the first intermediate (snail) host, thus

a

Simple parasites: growth or reproduction
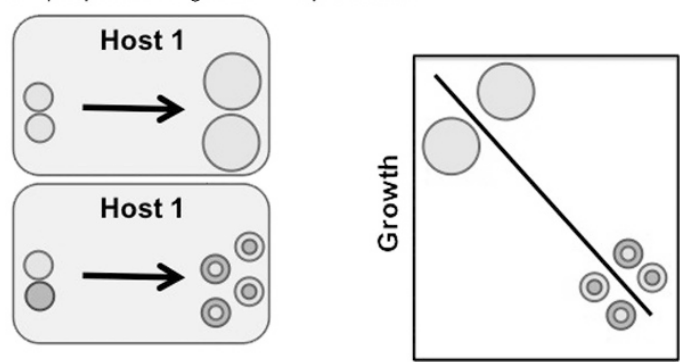

Reproduction

b

Complex parasites: escaping the growth-reproduction trade-off
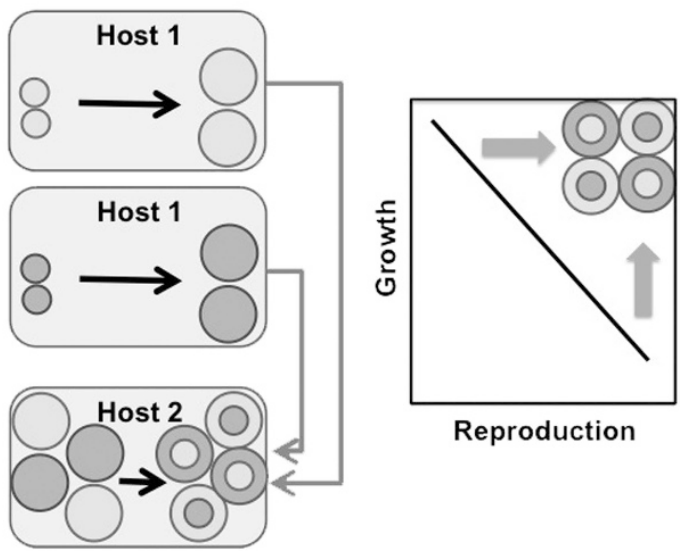

Reproduction

Figure 1 Growth-reproduction trade-off for SLPs and CLPS. Solid blue or solid orange circles represent two different clonal genotypes; circles that are both blue and orange represent the sexual progeny resulting from mating events between the blue and orange genotypes. (a) Within-host competition subjects simple parasites to the growth-reproduction trade-off: asexual reproduction leads to a few large parasites, whereas sexual reproduction leads to more small parasites. (b) CLPs can escape this trade-off: both growth and reproduction can, to a greater extent, evolve independently (see light grey arrows). A full color version of this figure is available at the Heredity journal online 
optimising growth by minimising parasite competition. Immature trematodes then go on to infect the second intermediate host (fish) along with other (genotypically different) trematodes. Finally, the definitive host (a bird) consumes numerous fish and thus collects many parasite genotypes, providing an arena for the many mature trematode genotypes to mix and mate (Rauch et al., 2005). Contrasting selection pressures from each host type could thus allow parasite life-history traits related to growth and reproduction to evolve and adapt independently. Indeed, some cestode genera exhibit complete separation of growth and reproduction: Schistocephalus, Ligula and Digramma sp. only feed and grow in their intermediate hosts, and only reproduce in the definitive host (Dubinina, 1980; Benesh et al., 2013); similarly, Plasmodium, Theileria and Toxoplasma sp. undergo asexual replication (equivalent to growth) in intermediate hosts, reproduce sexually in only their definitive hosts (Roos, 2005). This has important implications for the virulence experienced by various hosts, because virulence often increases with either parasite size or density.

The evolution of a complex lifecycle causes shifts in selection pressures on different life history stages and we acknowledge that the complete separation of growth and reproduction according to host type is not a universal feature among CLPs. For example, mortality rates are often lower in definitive hosts than in intermediate hosts (Parker et al., 2003), resulting in selection for growth in the definitive host before reproduction occurs (Parker et al., 2003; Iwasa and Wada, 2006; Benesh et al., 2013). So parasite schedules of investment in growth and reproduction (and resultant changes in virulence) can be both a cause and a consequence of an increase in lifecycle complexity.

\section{CLPS AND HOST-PARASITE COEVOLUTION}

CLPs often infect very different host types over the course of their lifecycle, for example, invertebrates and vertebrates. Moreover, many CLPs have at least one highly mobile host that promotes gene flow among parasite populations (Jarne and Théron, 2001). Such gene flow can potentially limit the ability of parasites to adapt to hosts and thus prevent specialisation on either or all host types (Slatkin, 1987; Lenormand, 2002). These breaks to adaptation may lead one to predict that parasites become less specialised as their lifecycles increase in complexity. This is not the case.

There are many examples of extreme host specialisation among CLPs: Plasmodium species specialise on different species of bird, mammal and reptile, and show little evidence for host switching (Perkins and Schall, 2002; Paul et al., 2003). Plasmodium falciparum even exhibits genetic specificity with its Anopheles gambiae (mosquito) hosts, where infection success depends on the exact combination of P. falciparum strain and mosquito genotype (Lambrechts et al., 2005). Schistosoma mansoni also shows evidence of genetic specificity with its snail intermediate host, Biomphalaria glabrata (Webster and Woolhouse, 1998; Davies et al., 2001; Mitta et al., 2012), and Microphallus trematodes exhibit adaptation to locally common genotypes of their snail intermediate hosts, Potamopygus antipodarum (Lively and Dybdahl, 2000; Dybdahl et al., 2008; Jokela et al., 2009).

\section{Patterns of local adaptation in CLPs}

How can a parasite have a complex lifecycle that frequently entails high between-population dispersal, and yet adapt to infect particular local genotypes of intermediate host, definitive host or both? Theory predicts parasites can show local adaptation (LA) to at least one host species (that is, achieve greater infection success of sympatric hosts than allopatric hosts), provided parasite migration rate exceeds host migration rate in a metapopulation (Gandon et al., 1996; Gandon and Michalakis, 2002; Figure 2). Put another way, the ability of parasites to adapt to their hosts (and vice versa) is contingent on their evolutionary potential, which is dependent on the supply of favourable alleles to the population (among other factors such as parasite genetic diversity and generation time; Gandon and Michalakis, 2002).

A recent meta-analysis found CLPs were no more likely to exhibit LA than SLPs (Greischar and Koskella, 2007). However, as discussed earlier, theory suggests that the presence of LA will crucially depend on the migration rate of the focal host species relative to the migration rate of the other host(s). This theory gives rise to the following hypotheses: (1) more mobile hosts will promote CLP dispersal, and thus increase the supply of novel genotypes for infection of less mobile hosts (sensu Jarne and Théron, 2001); (2) CLPs will exhibit LA to their least mobile host species; and (3) CLPs will exhibit either no LA or local maladaptation to their most mobile host.

Empirical studies provide considerable support for these hypotheses. Keeney et al. (2009) found that the trematodes Maritrema novozealandensis and Philopthalmus sp. exhibited higher withinpopulation genetic diversity and greater gene flow than their low mobility marine snail host, Zeacumantus subcarinatus. Dybdahl and Lively (1996) found Microphallus trematodes had much lower between-population genetic structure than their intermediate $P$. antipodarum (snail) hosts. Wild Schistostoma mansoni trematodes also had population genetic signatures of dispersal similar to those of their definitive rat hosts (and greater than their snail intermediate hosts: Prugnolle et al., 2005), and S. mansoni that infect snails, salmon and fish-eating birds have higher dispersal and less population structure than those which infect snails and salmon only (Criscione and Blouin, 2004). These four studies suggest the more mobile definitive hosts maintain genetic variation in parasite populations, supporting hypothesis (1). Microphallus also exhibits strong LA to sympatric snail populations (Lively et al., 2004; Dybdahl et al., 2008), and $S$. mattheei is locally adapted to its less mobile snail host Bulinus

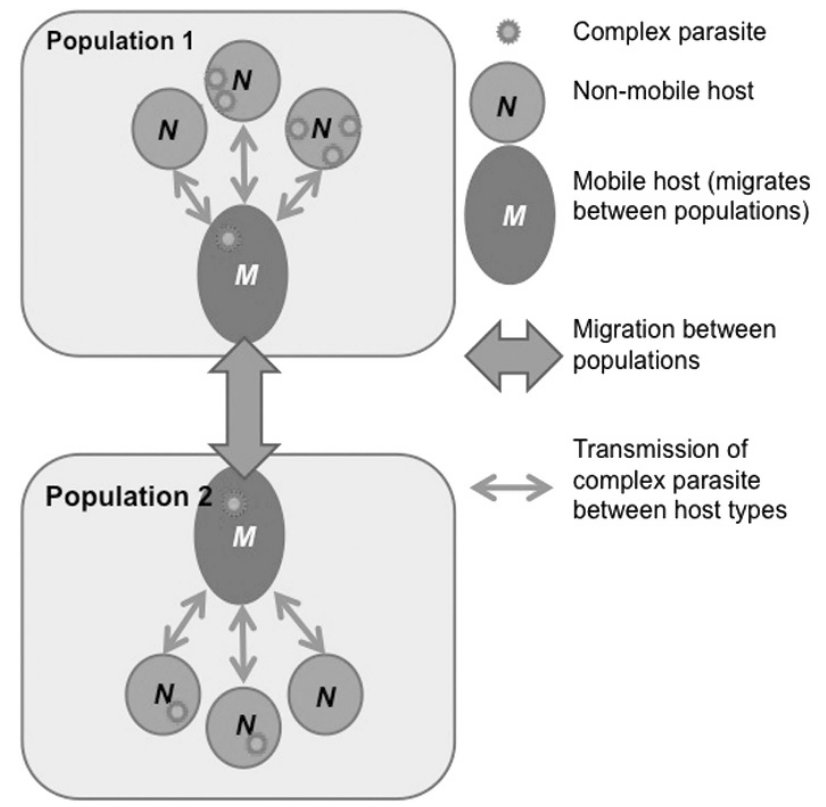

Figure 2 The evolutionary consequences of definitive host migration for a CLP metapopulation. Migration rates often vary among the different hosts of a CLP. The migration rate of a CLP is expected to be most similar to that of its most mobile host-in this case the definitive host (Jarne and Théron, 2001). Immigration of mobile hosts into a population can supply novel parasite genotypes, promoting parasite LA to the less-mobile host (Gandon et al., 1996; Gandon and Michalakis, 2002). 
globosus (Manning et al., 1995), consistent with hypothesis (2). Conversely, Prugnolle et al. (2006) found no evidence for S. mansoni LA to the snail (Biomphalaria glabrata) in a population where S. mansoni and B. glabrata were found to have similar migration rates. Diplostomum pseudopathaceum trematodes demonstrated local maladaptation to their more mobile fish hosts (Gasterosteus aculeatus) and a haemogregarine parasite (phylum Sporozoa) showed local maladaptation to populations of its most mobile host, the lizard Gallotia galloti (Oppliger et al., 1999). These three studies support hypothesis (3). It is, however, important to note that many studies of LA in CLP focus on trematodes. Further study of other CLP taxa is required to effectively test whether CLPs are generally more likely to exhibit LA to their less mobile hosts.

For coevolutionary systems that rely on allelic novelty, the supply of allelic diversity to a population may accelerate evolutionary change in parasite populations by contributing alleles that evade prevailing host immune responses. Conversely, if host migration rate exceeds that of the parasite in a metapopulation, the host may have the upper hand, as higher host migration could fuel the evolution of host resistance to sympatric parasites (Gandon et al., 1996, but see also Lively, 1999; Gandon and Michalakis, 2002). The potential of high-dispersal hosts to spread genotypes of CLPs and thus fuel parasite LA could pose major problems for the management of many human and livestock diseases. Limiting vector dispersal may therefore be an important component of disease management.

\section{MECHANISMS OF INFECTION AND HOST IMMUNE EVASION IN CLPS: DIFFERENT MECHANISMS FOR DIFFERENT HOSTS?}

Do CLPs use the same or different machinery to infect each host type? Plasmodium berghei, P. chabaudi and P. yoelli, as well as the filarial worm Brugia malayi exhibit different patterns of gene expression when in mosquitoes than when they are in their mammal hosts (Hall et al., 2005; Lasonder et al., 2008; Choi et al., 2011). The Lyme disease bacterium Borrelia burgdorferi also expresses different genes when infecting ticks and mammals (Revel et al., 2002; Pal and Fikrig, 2003; Rogers et al., 2009). These findings have been interpreted to indicate that the parasite uses distinct infection mechanisms to infect each host type. However, in the absence of functional knowledge, observations of differential gene expression in different hosts could merely reflect changes in parasite developmental stage, rather than host-specific infection mechanisms.

Whole-organism study has provided considerable insight into the overlap between infection mechanisms. The ability of the helminth Schistosoma mansoni to successfully infect its intermediate snail host trades-off with success at infecting its definitive mouse host (Davies et al., 2001). This suggests some of the genes associated with infecting snails may also influence infection success in mice and that antagonistic pleiotropic effects could maintain allelic diversity in these genes (Webster and Woolhouse, 1998; Davies et al., 2001; Webster et al., 2007; Mitta et al., 2012). The tapeworm, Schistocephalus solidus exhibits a slightly different trade-off between its ability to overcome the innate immune system (present in both invertebrates and vertebrates) and the acquired immune system (present in vertebrates only; Hammerschmidt and Kurtz, 2005).

Studies of the molecular mechanisms of infection have further added to our understanding of CLPs. Differences in gene expression in the Lyme disease-causing bacterium, Borrelia burgdorferi are intrinsically linked with distinct mechanisms for infecting intermediate hosts (deer ticks, Ixodes sp.) and definitive (vertebrate) hosts. Borrelia expresses different suites of genes as it migrates to different tissues within the tick (Revel et al., 2002; Rogers et al., 2009); upregulation of
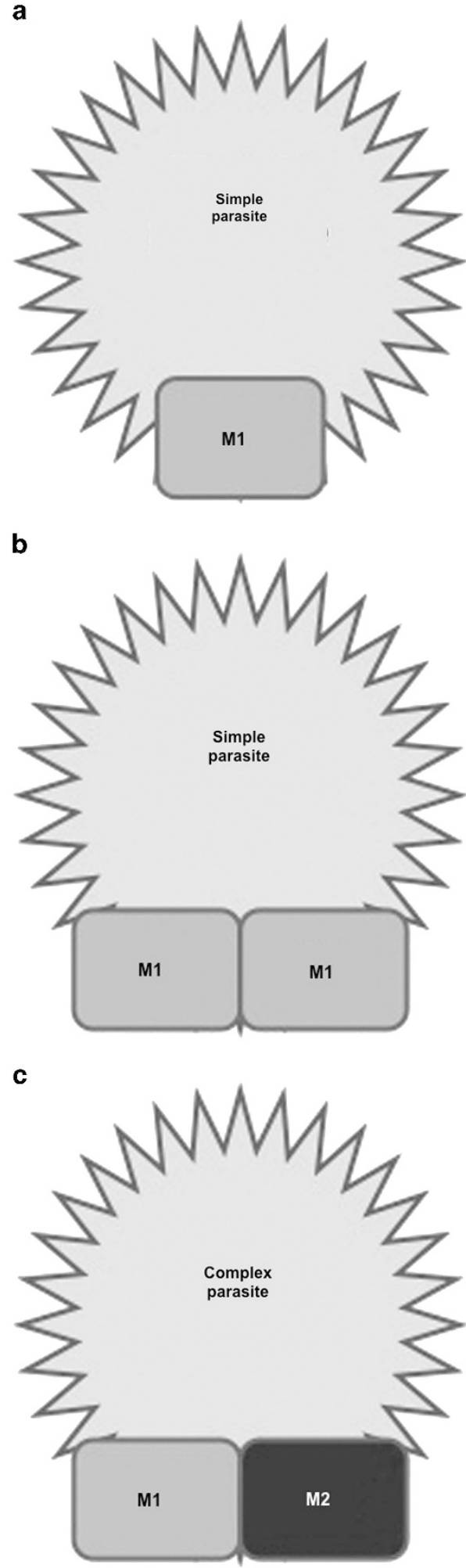

Figure 3 Scenario for the evolution of host-specific infection mechanisms with increasing parasite lifecycle complexity. (a) The ancestral state: a parasite with a single infection mechanism (M1) for infecting different host types. (b) Gene duplication creates paralogues of the ancestral infection mechanism: a precursor for distinct infection mechanisms. (c) Varying selective pressures from intermediate and definitive hosts lead to neofunctionalisation in one of the paralogues (M2) and the evolution distinct infection mechanisms. 
the $o s p A$ gene promotes exit from the tick gut and $\operatorname{sp} p$ is required for passage to the tick salivary gland and is essential for early infection in the vertebrate host (Gilmore and Piesman, 2000; Tilly et al., 2006). Borrelia also blocks the vertebrate complement system by producing Erp (OspE/F-like Related Protein) proteins on its cell surface (Stevenson et al., 2002) and uses the saliva of its tick host as an additional shield against the vertebrate immune system (tick saliva inactivates the complement system and therefore inhibits the capacity of vertebrate neutrophils to phagocytose microbial parasites; Ribiero et al., 1990).

Plasmodium also uses different infection mechanisms for vertebrate and invertebrate hosts (Baum et al., 2008): both ookinetes (the mosquito-infecting life stage) and sporozoites (the mammalinfecting life stage) express genes from the TRAP-like gene family (thrombospondin-related anonymous protein), which mediate host cell recognition (Sultan et al., 1997; Matuschewski et al., 2002). The MAOP protein (membrane attack ookinete protein; a type of TRAP) is required for entry into mosquito midgut cells (Kadota et al., 2004) and the closely related SPECT1 and SPECT2 proteins (sporozoite micronemal protein essential for cell transversal) are required for successful infection of mammalian liver hepatocytes (Yuda and Ishino, 2004).

How could separate mechanisms for infecting different host types have evolved? The degree of overlap between infection mechanisms used to infect intermediate and definitive hosts can tell us a great deal about the evolution of lifecycle complexity in parasites, especially as infection mechanisms are likely to be linked with parasite developmental stage (Aly et al., 2009). Evidence from both Borrelia and Plasmodium (from Osp genes and TRAP genes, respectively) suggest that relatively recent gene duplication events have generated multiple paralogues, resulting in the evolution of two infection mechanisms from a single ancestral mechanism (Revel et al., 2002; Pal and Fikrig, 2003; Aly et al., 2009; see Figure 3). If the scenario outlined in Figure 2 holds, one expects that knowledge of the infection mechanisms used for different hosts may allow characterisation of the degree of parasite specialisation and assessment of the potential for evolutionary shifts in lifecycle.

\section{Testing the gene duplication hypothesis and uncovering genetic signatures of coevolution}

We suggest gene duplication may be a common mechanism for the acquisition of novel hosts during the evolution of a complex parasite lifecycle. Examination of molecular evolution in infection gene paralogues will help address essential questions in CLP biology, for example, whether particular lifecycle complexity evolved through UI or DI. Where acquisition of a novel host has been associated with gene duplication, one paralogue will have retained its function as an infection mechanism for the ancestral host, whereas the second locus will have been subjected to strong selection for neofunctionalisation (and will show evidence of elevated adaptive substitution) as the parasite adapts to its new additional host. By looking for evidence of duplication and neofunctionalisation among genes associated with infecting different host types, one can uncover whether lifecycle complexity evolved through UI or DI. Increased lifecycle complexity likely evolved via UI if the neofunctionalised paralogue is associated with infection of the definitive host, whereas DI is more likely to have occurred if the neofunctionalised paralogue is associated with infection of the intermediate host (Box 1).

Another application of sequence evolution studies would be to test the prediction that antagonistic host-parasite coevolution should be strongest between the parasite and its least mobile host. Strong host-parasite coevolution may be evident as the maintenance of divergent genotypes through negative frequency selection, or as the depletion of genetic diversity because of recurrent selective sweeps. Comparing sequence evolution in paralagous gene loci with similar infection functions could provide a strong opportunity to test the relative departures from neutrality in these two loci and hence whether the parasite has the most dynamic coevolutionary interaction with its intermediate or definitive host.

\section{CONCLUSION}

Understanding the factors that promote and maintain complex lifecycles in parasites is important for both basic and applied biology, as so many disease-causing organisms have a complex lifecycle. Theory has advanced our understanding of the selective pressures that may have favoured increased complexity in the lifecycle of individual parasite species. The empirical study of parasites that facultatively alter their lifecycle complexity can help us understand the ecological circumstances where lifecycle complexity benefits parasite fitness, and how a more complex parasite lifecycle affects virulence towards various hosts (Box 1). Genetic, genomic and post-genomic approaches have also improved our knowledge of infection mechanisms in CLPs (especially in B. burgdorferi and P. falciparum), but they need to be extended (see Box 1). What are the genes determining variation in ability to infect intermediate and definitive hosts? Is there overlap between the mechanisms for infecting each host type? Answering these questions in different CLP-host systems is an important goal. Together, these theoretical, phenomenological and mechanistic approaches will foster a more general understanding of the causes and consequences of increased complexity in parasite lifecycles.

\section{DATA ARCHIVING}

There were no data to deposit.

\section{CONFLICT OF INTEREST}

The authors declare no conflict of interest.

\section{ACKNOWLEDGEMENTS}

SKJRA is funded by a University of Stirling Impact Fellowship and a NERC Independent Research Fellowship (NE/L011549/1). We are grateful to Stéphane Cornet, Meghan Duffy, Andrew Dobson and two anonymous reviewers for comments.

Aly ASI, Vaughan AM, Kappe SHI (2009). Malaria parasite development in the mosquito and infection of the mammalian host. Ann Rev Microbiol 63: 195-221.

Anderson RC (2000). Nematode Parasites of Vertebrates: Their Development and Transmission. CABI Publishing: Wallingford, UK.

Balmer O, Stearns SC, Schötzau A, Brun R (2009). Intraspecific competition between co-infecting parasite strains enhances host survival in African trypanosomes. Ecology 90: 3367-3378.

Baum J, Gilberger T-W, Frischknecht F, Meissner M (2008). Host-cell invasion by malaria parasites: insights from Plasmodium and Toxoplasma. Trends Parasitol 24: 557-563.

Bell AS, De Roode JC, Sim D, Read AF (2006). Within-host competition in genetically diverse malaria infections: parasite virulence and competitive success. Evolution 60 1358-1371.

Benesh DP, Chubb JC, Parker GA (2013). Complex life cycles: why refrain from growth before reproduction in the adult niche? Am Naturalist 181: 39-51.

Brown SP, Renaud F, Guegan J-F, Thomas F (2001). Evolution of trophic transmission in parasites: the need to reach a mating place. J Evol Biol 14: 815-820.

Choi Y-J, Ghedin E, Berriman M, McQuillan J, Holroyd N, Mayhew GF et al. (2011). A deep sequencing approach to comparatively analyze the transcriptome of lifecycle stages of the filarial worm, Brugia malayi. PLOS Negl Trop Dis 5: e1409.

Choisy M, Brown SP, Lafferty KD, Thomas F (2003). Evolution of trophic transmission in parasites: why add intermediate hosts. Am Naturalist 162: 172-181.

Christen M, Milinski M (2003). The consequences of self-fertilization and outcrossing of the cestode Schistocephalus solidus in its second intermediate host. Parasitology 126: 369-378 
Cornet S (2011). Density-dependent effects on parasite growth and parasite-induced host immunodepression in the larval helminth Pomphorhynchus laevis. Parasitology 138: 257-265.

Cribb TH, Bray RA, Olson PD, Littlewood DTJ (2003). Life cycle evolution in the Digenea: a new perspective from phylogeny. Adv Parasitol 54: 197-254.

Criscione CD, Blouin MS (2004). Life cycles shape parasite evolution: comparative population genetics of salmon trematodes. Evolution 58: 198-202.

Crompton DWT, Nickol BB (1985). Biology of the Acanthocephala. Cambridge University Press: Cambridge, UK.

Davies CM, Webster JP, Woolhous ME (2001). Trade-offs in the evolution of virulence in an indirectly transmitted macroparasite. Proc $R$ Soc B 268: 251-257.

De Roode JC, Pansini R, Cheesman SJ, Helinski MEH, Huijben S, Wargo AR et al. (2005). Virulence and competitive ability in genetically diverse malaria infections. Proc Nat/ Acad Sci USA 102: 7624-7628.

Dianne L, Bollache L, Lagrue C, Franceschi N, Rigaud T (2012). Larval size in acanthocephalan parasites: influence of intraspecific competition and effects on intermediate host behavioural changes. Parasites Vectors 5: 1-7.

Dubey JP (1977). Toxoplasma, Hammondia, Besnoitia, Sarcocystis, and other tissue cystforming coccidia of man and animals. In: Kreier JP (ed) Parasitic Protozoa. Academic Press: New York. pp 101-237.

Dubinina MN (1980). Tapeworms (Cestoda, Ligulidae) of the Fauna of the USSR. Amerind: New Delhi, India.

Dybdahl MF, Jokela J, Delph LF, Koskella B, Lively CM (2008). Hybrid fitness in a locally adapted parasite. Am Naturalist 172: 772-782.

Dybdahl MF, Lively CM (1996). The geography of coevolution: comparative population structures for a snail and its trematode parasite. Evolution 50: 2264-2275.

Gandon S (2004). Evolution of multihost parasites. Evolution 58: 455-469.

Gandon S, Capowiez Y, Dubois Y, Michalakis Y, Olivieri I (1996). Local adaptation and gene-for-gene coevolution in a metapopulation model. Proc $R$ Soc Lond B Biol Sci 263: 1003-1009.

Gandon S, Michalakis Y (2002). Local adaptation, evolutionary potential and host-parasite coevolution: interactions between migration, mutation, population size and generation time. J Evol Biol 15: 451-462.

Gilmore RD, Piesman J (2000). Inhibition of Borrelia burgdorferi migration from the midgut to the salivary glands following feeding by ticks on OspC-immunized mice. Infect Immun 58: 411-414.

Grabda-Kazubska B (1976). Abbreviation of the life cycles in plagiorchid trematodes: general remarks. Acta Parasitologica Polonica 24: 125-141.

Greischar MA, Koskella B (2007). Asynthesis of experimental work on parasite local adaptation. Ecol Lett 10: 418-434

Hall N, Karras M, Raine JD, Carlton JM, Kooij TWA, Berriman M et al. (2005). A comprehensive survey of the Plasmodium life cycle by genomic, transcriptomic, and proteomic analyses. Science 307: 82-86.

Hammerschmidt K, Koch K, Milinski M, Chubb JC, Parker GA (2009). When to go: optimization of host switching in parasites with complex life cycles. Evolution 63 1976-1986.

Hammerschmidt K, Kurtz J (2005). Surface carbohydrate composition of a tapeworm in its consecutive intermediate hosts: individual variation and fitness consequences. Int $\mathrm{J}$ Parasitol 35: 1499-1507.

House PK, Vyas A, Sapolsky R (2011). Predator cat odors activate sexual arousal pathways in brains of Toxoplasma gondii infected rats. PLOS ONE 6: e23277.

Iwasa Y, Wada G (2006). Complex life cycle and body sizes at life-history transmitions for macroparasites. Evol Ecol Res 8: 1427-1443.

Jäger I, Schjørring S (2006). Multiple infections: relatedness and time between infections affect the establishment and growth of the cestode Schistocephalus solidus in its stickleback host. Evolution 60: 616-622.

Jarne $P$, Théron A (2001). Genetic structure in populations of flukes and snails: a practical approach and review. Parasitology 123: S27-S40.

Jokela J, Dybdahl M, Lively CM (2009). The maintenance of sex, clonal dynamics, and host-parasite coevolution in a mixed population of sexual and asexual snails. Am Naturalist 174: S43-S53.

Kadota K, Ishino T, Matsuyama T, Chinzei M, Yuda M (2004). Essential role of membraneattack protein in malarial transmission to mosquito host. Proc Natl Acad Sci USA 101: $16310-16315$

Keeney DB, King T, Rowe DL, Poulin R (2009). Contrasting mtDNA diversity and population structure in a direct-developing marine gastropod and its trematode parasites. Mol Ecol 18: 4591-4603.

Lagrue C, Poulin R (2007). Life cycle abbreviation in the trematode Coitocaecum parvum: can parasites adjust to variable conditions? J Evol Biol 20: 1189-1195.

Lagrue C, Poulin R (2008). Intra- and inter-specific competition among helminth parasites: effects on Coitocaecum parvum life-history strategy, size and fecundity. Int J Parasitol 38: 1435-1444.

Lagrue C, Waters JM, Poulin R, Keeney DB (2007). Microsatellite loci for the progenetic trematode, Coitocaecum parvum (Opercoelidae). Mol Ecol Notes 7: 694-696.

Lambrechts L, Halbert J, Durand P, Gouagna LC, Koella JC (2005). Host genotype by parasite genotype interactions underlying the resistance of anopheline mosquitoes to Plasmodium falciparum. Malaria J 4: 3

Lasonder E, Janse CJ, van Gemert G-J, Mair GR, Vermunt AMW, Douradinha BG et al. (2008). Proteomic profiling of Plasmodium sporozoite maturation identifies new proteins essential for parasite development and infectivity. PLoS Pathogens 4: e1000195.
Lefebvre F, Poulin R (2005). Alternative reproductive strategies in the progenetic trematode Coitocaecum parvum: comparison of selfing and mating worms. J Parasito 91: 93-98.

Lenormand T (2002). Gene flow and the limits to natural selection. Trends Ecol Evol 17: $183-189$.

Lively CM (1999). Migration, virulence, and the geographic mosaic of adaptation by parasites. Am Naturalist 153: S34-S47.

Lively CM, Dybdahl ME, Jokela J, Osnas EE, Delph LE (2004). Host sex and local adaptation by parasites in a snail-trematode interaction. Am Naturalist 164: S6-S18.

Lively CM, Dybdahl MF (2000). Parasite adaptation to locally common host genotypes. Nature 405: 679-681.

Manning SD, Woolhouse MEJ, Ndamba J (1995). Geographic compatibility of the freshwater snail Bulinus globosus and schistosomes from the Zimbabwe highveld. Int J Parasitol 25: 37-42.

Matuschewski K, Nunes AC, Nussenzweig V, Menard R (2002). Plasmodium sporozoite invasion into insect and mammalian cells is directed by the same dual binding system. EMBO J 21: 1597-1606.

Michaud M, Milinski M, Parker GA, Chubb JC (2006). Competitive growth strategies in intermediate hosts: experimental tests of a parasite life-history model using the cestode Schistocephalus solidus. Evol Ecol 20: 37-57.

Minot S, Melo MB, Li F, Lu D, Niedelman W, Levine SS et al. (2012). Admixture and recombination among Toxoplasma gondii lineages explain global genome diversity. Proc Natl Acad Sci USA 109: 13458-13463.

Mitta G, Adema CM, Gourbal B, Loker ES, Theron A (2012). Compatibility polymorphism in snail/schistosome interactions: from field to theory to molecular mechanisms. Dev Comp Immunol 37: 1-8.

Morand S, Robert F, Connors VA (1995). Complexity in parasite life cycles: population biology of cestodes in fish. J Animal Ecol 64: 256-264.

Nasincova V, Scholz T (1994). The life cycle of Asymphylodora tincae (Modeer 1790) (Trematoda: Monorchiidae): a unique development in monorchiid trematodes. Parasito Res 80: 192-197.

Oppliger A, Vernet R, Baez M (1999). Parasite local maladaptation in the Canarian lizard Gallotia galloti (Reptilia: Lacertidae) parasitized by haemogregarian blood parasite. J Evol Biol 12: 951-955.

Orlofske SA, Jadin RC, Preston JL, Johnson PTJ (2012). Parasite transmission in complex communities: predators and alternative hosts alter pathogenic infections in amphibians. Ecology 93: 1247-1253.

Pal U, Fikrig E (2003). Adaptation of Borrelia burgdorferi in the vector and vertebrate host. Microbes Infect 5: 659-666.

Park J-K, Kim K-H, Kang S, Kim W, Eom KS, Littlewood DTJ (2007). A common origin of complex life cycles in parasitic flatworms: evidence from the complete mitochondrial genome of Microcotyle sebastis (Monogenea: Platyhelminthes), BMC Evol Biol 7: 11.

Parker GA, Chubb JC, Ball MA, Roberts GN (2003). Evolution of complex life cycles in helminth parasites. Nature 425: 480-484.

Paul REL, Ariey F, Robert V (2003). The evolutionary ecology of Plasmodium. Ecol Lett 6: 866-880.

Perkins SL, Schall JJ (2002). A molecular phylogeny of malarial parasites recovered from cytochrome $b$ gen sequences. J Parasitol 121: 972-978.

Poulin R (1995). "Adaptive" changes in the behaviour of parasitized animals: a critical review. Int J Parasito/ 25: 1371-1383.

Poulin R (2003). Information about transmission opportunities triggers a life-history switch in a parasite. Evolution 57: 2899-2903.

Poulin R, Cribb TH (2002). Trematode life cycles: short is sweet? Trends Parasitol 18 : $176-183$.

Prugnolle F, Théron A, Pointier JP, Jabbour-Zahab R, Jarne P, Durand P et al. (2005). Dispersal in a parasitic worm and its two hosts: consequence for local adaptation. Evolution 59: 296-303.

Prugnolle F, De Meeûs T, Pointier JP, Durand P, Rognon A, Théron A (2006). Geographical variations in infectivity and susceptibility in the host-parasite system Schistosoma mansoni/Biomphalaria glabrata: no evidence for local adaptation. Parasitology 133: 313-319.

Rauch G, Kalbe M, Reusch TBH (2005). How a complex life cycle can improve a parasite's sex life. J Evol Biol 18: 1069-1075.

Revel AT, Talaat AM, Norgard MV (2002). DNA microarray analysis of differential gene expression in Borrelia burgdorferi, the Lyme disease spirochete. Proc Natl Acad Sci USA 99: $1562-1567$

Ribiero JM, Weis JJ, Telford S III (1990). Saliva of the tick Ixodes dammini inhibits neutrophil function. Exp Parasitol 70: 380-388.

Riley J (1983). Recent advances in our understanding of pentastomid reproductive biology. Parasitology 86: 59-83.

Robert F, Renaud F, Mathieu E, Gabrion C (1988). Importance of the paratenic host in the biology of Bothriocephalus gregarius (Cestoda: Pseudophyllidea), a parasite of the turbot. Int J Parasitol 18: 611-621.

Rogers EA, Terekhova D, Zhang H-M, Hovis KM, Schwartz I, Marconi RT (2009). Rrp1, a cyclic-di-GMP-producing response regulator, is an important regulator of Borrelia burgdorferi core cellular functions. Mol Microbiol 71: 1551-1573.

Roos DS (2005). Themes and variations in apicomplexan parasite biology. Science 309 : 72-73

Slatkin M (1987). Gene flow and the geographic structure of natural populations. Science 236: 787-792.

Stevenson B, El-Hage N, Hines NA, Miller JC, Babb K (2002). Differential binding of host complement inhibitor factor $\mathrm{H}$ by Borrelia burgdorferi Erp surface proteins: a possible 
mechanism underlying the expansive host range of Lyme disease spirochetes. Infect Immun 70: 491-497.

Su C, Evans D, Cole RH, Kissinger JC, Ajioka JW, Sibley LD (2003). Recent expansion of Toxoplasma through enhanced oral transmission. Science 299 414-416.

Sultan AA, Thathy V, Frevert U, Robson KJH, Crisanti A, Nussenzweig V et al. (1997). TRAP is necessary for gliding motility and infectivity of Plasmodium sporozoites. Cell 90: 511-522.

Tilly K, Krum JG, Bestor A, Jewett MW, Grimm D, Bueschel D et al. (2006). Borrelia burgdorferi OspC protein required exclusively in a crucial early stage of mammalian infection. J Immunol 74: 3554-3564.

Tyler KM, Engman DM (2001). The life cycle of Trypanosoma cruzi revisited. Int J Parasitol 31: 472-481.

Vickerman K, Tetley L, Hendry KAK, Turner CMR (1988). Biology of African trypanosomes in the tsetse fly. Biol Cell 64: 109-119.

Wang CL, Thomas F (2002). Egg production by metacercariae of Microphallus papillorobustus: a reproductive insurance? J Helminthol 76: 279-281.

Webster JP, Shrivastava J, Johnson P, Blair L (2007). Is host-schistosome coevolution going anywhere? BMC Evol Biol 7: 91.
Webster JP, Woolhouse MEJ (1998). Selection and strain specificity of compatibility between snail intermediate hosts and their parasitic schistosomes. Evolution 52 1627-1634.

Wedekind C, Strahm D, Strärer L (1998). Evidence for strategic egg production in a hermaphroditic cestode. Parasitology 117: 373-382.

Yuda M, Ishino T (2004). Liver invasion by malarial parasites-how do malarial parasites break through the host barrier? Cell Microbiol 6: 1119-1125.

Attribution 4.0 International License. The images or other third party material in this article are included in the article's Creative Commons license, unless indicated otherwise in the credit line; if the material is not included under the Creative Commons license, users will need to obtain permission from the license holder to reproduce the material. To view a copy of this license, visit http:// creativecommons.org/licenses/by/4.0/ 